\title{
Mesenchymal stromal cell therapy in ischemic stroke
}

\section{Ye Zhang \\ Hong Deng \\ Chao Pan \\ Yang $\mathrm{Hu}$ \\ Qian Wu \\ $\mathrm{Na}$ Liu \\ Zhouping Tang}

Department of Neurology, Tongji Hospital, Tongji Medical College, Huazhong University of Science and Technology, Wuhan, Hubei, People's Republic of China
Correspondence: Zhouping Tang Department of Neurology, Tongji Hospital, to Tongji Medical College, Huazhong University of Science and Technology, Jiefang Blvd., No. 1095 , Wuhan 430030, People's Republic of China

Tel +86278366 3337

Fax +862783662639

Email ddjtzp@163.com
This article was published in the following Dove Press journal:

Journal of Neurorestoratology

10 November 2016

Number of times this article has been viewed

Abstract: Stroke is a clinical disease with high incidence, high disability rate, and high mortality. But effective and safe therapy for stroke remains limited. Adult mesenchymal stromal cells (MSCs) perform a variety of therapeutic functions. MSC delivery improves neurological outcomes in ischemic stroke models via neurorestorative and neuroprotective effects such as angiogenic effects, promoting endogenous proliferation, and reducing apoptosis and inflammation. MSC secretome also showed powerful therapeutic effects as a cell-based therapy in animal experiments. Several clinical trials on MSC implantation via different routes have now been completed in patients with stroke. Although challenges such as immunogenicity of alloMSCs and large-scale production strategies need to be overcome, MSCs can be considered as a promising potential therapy for ischemic stroke.

Keywords: mesenchymal stromal cell, stroke, therapy, transplantation, exosomes

\section{Introduction}

Stroke is the second leading cause of death worldwide resulting in around six million deaths every year. A stroke attack happens in over 15 million people every year and leaves five million people disabled. ${ }^{1}$ In spite of decades of research and clinical trials, therapeutic approaches remain limited. Searching for safe and effective therapies is urgently required. Mesenchymal stromal cells (MSCs) are adult progenitor cells with multipotent and self-renewing capacity that can be easily obtained from numerous sources including bone marrow, umbilical cord, adipose tissue, and dental pulp. Their self-renewal is similar to that of embryonic stem cells because of the shared expression of some pluripotency genes such as $N A N O G$ and Sox $2 .^{2}$ The International Society for Cellular Therapy has proposed four criteria to define MSCs. These are: 1) plastic adherence in standard culture conditions; 2) fibroblast-like morphology; 3) specific surface antigen expression: low expression $(<2 \%+)$ of hematopoietic markers CD19, CD34, CD14, CD11b or CD45, CD79a, and HLA-DR and vascular marker CD31 as well as high expression (>95\%+) of CD105, CD73, and CD90; and 4) in vitro differentiation potential to give rise to osteoblasts, adipocytes, and chondroblasts. ${ }^{3} \mathrm{MSC}$ are numerically the most favored cell type in stem cell therapy presently being studied in clinical trials. ${ }^{4}$ Therapeutic effects in ischemic stroke following MSC transplantation have been reported in many animal experiments and in some clinical trials. In this review, we elaborate on the therapeutic effects of transplanted MSCs in ischemic stroke models and illustrate clinical studies focusing on MSC therapy in stroke. 


\section{Cell-based therapy in preclinical ischemic stroke model}

Previously, MSCs have shown promising efficacy in ischemic stroke model. Implantation of rodent or human MSCs by different means, including systemically and by intracerebral transplantation, several hours to days after cerebral ischemia has been shown to reduce infarct size and improve neurological function in rodent ischemia stroke models through its neurorestorative and neuroprotective effects. ${ }^{5,6,7}$ MSCs delivered systemically (intravenously or intra-arterially) can pass the blood-brain barrier (BBB), enter the brain, and localize to sites of injury, inflammation, and ischemia in spite of that most of them are stuck in the lung vasculature. ${ }^{5,6} \mathrm{Kim}$ et $\mathrm{al}^{8}$ had confirmed MSCs' brain tropism by performing whole-body imaging of radiolabeled human adipose-derived MSCs systemically given to rats with middle cerebral artery occlusion (MCAO). MSCs were trapped in the lungs for the first 2 hours after stroke onset but continued migrating over time to be found within the region of ischemic lesions. When MSCs were administered intravenously following a stroke, an increased number of MSCs labeled with BrdU was seen in the injured hemisphere than in the noninjured side. Increase in astrocytosis, vascularization, and endogenous proliferation were also reported. ${ }^{9-11}$ Adipose-derived MSC administration after transient global cerebral ischemia resulted in a significant protective effect against hippocampal neuronal death, which might be linked to the preservation of BBB integrity, prevention of endothelial damage, and a decrease in neutrophil infiltration. ${ }^{12}$

Moisan et $\mathrm{l}^{13}$ found enhanced vascular density in the ischemic area after intravenous injection of clinical-grade human MSCs in experimental stroke and observed that this was through the release of endogenous angiogenic factors such as VEGF, angiogenin-1, and TGF- $\beta 1$ enhancing the stabilization of newly formed vessels. Inflammation was also significantly suppressed by umbilical cord-derived MSC delivery as indicated by elevated levels of anti-inflammatory cytokines IL-4 and TGF- $\beta$ and lower expression of IL-1 $\beta$ and TNF- $\alpha .{ }^{14}$

MSC transplantation at an earlier time after MCAO resulted in better functional recovery, and the preferential migration of MSCs to the cortex, as displayed in the earlytransplant group, may be an explanation for this. A further reasoning for this was that the time-dependent expression of MCP-1 and stromal cell-derived factor-1 between ischemic regions induces the different migration of MSCs. Highest expression of MCP-1 one day after stroke may mediate preferential migration of MSCs to the cortex, thus leading to enhanced improvement. ${ }^{15}$ Delayed transplantation of MSCs also showed encouraging improvement in neurological function after stroke, and this is more critical in terms of clinical utility. ${ }^{16}$

Dynamic regulation of metabolites plays an important role in stroke. For various pathological conditions, polyamines (PAs) act as an important biochemical indicator in metabolic pathways. An investigation of PA level changes showed that putrescine, cadaverine, and spermidine in brain tissues of human bone marrow-derived MSCs treatment group were significantly reduced in comparison to controls. This study proved that MSC transplantation could ameliorate PA metabolic dysfunction in ischemic brain injuries. ${ }^{17}$

Cheng et $\mathrm{al}^{18}$ demonstrated that human bone marrowderived MSC treatment via tail vein injection within 30 minutes after stroke ameliorated neurological deficit, brain edema, and infarct volume via a mechanism involving increasing TGF- $\beta$ level modulating peripheral immunoinflammation. Previous studies also observed that MSCs reduce the brain water content and protect $\mathrm{BBB}$ integrity following cerebral ischemia. ${ }^{12,13}$ Levels of AQP-4 and IL-1 $\beta$ were also considerably reduced. ${ }^{7}$ More recently, Tang et a ${ }^{19}$ showed that human bone marrow-derived MSCs retained their therapeutic efficacy on maintaining BBB integrity through the reduction of astrocyte apoptosis, and this appeared to be due to the attenuated inflammatory response and downregulated AQP4 expression through p38-MAPK signaling pathway.

Several studies demonstrated the potential of gene overexpression strategies to enhance MSC efficacy with genes such as BDNF, Notch-1, VEGF, and CXCR4.$^{20,21}$ Recently, MSCs with genetically modified CXCR4, a surface adhesion molecule, showed enhanced migration activity and enhanced neuroprotection and angiogenesis effects in the MCAO model. ${ }^{21}$ Jeong et al, ${ }^{20}$ in an in vivo experiment, showed that treatment with MSCs overexpressing BDNF, which is secreted by brain cells and induces neuroprotection, caused an enhanced proliferation of endogenous neural stem cells and suppression of cell death. They also observed that more neuroblasts and mature neurons differentiated from newly formed cells in the subventricular zone and the surrounding ischemic area. Finally, delivery of human bone marrowderived MSCs overexpressing BDNF and Noggin, which induces neuronal differentiation, decreased inflammation and apoptosis to a greater extent than control MSCs through the Akt/guanosine kinase-3 $\beta$ and TLR4/MyD88 pathways in an ischemic stroke model. ${ }^{22}$ 
Another discovery is that hypoxia-preconditioned MSCs promoted neurological functional recovery via enhancing significantly the cell's homing to the injured area and neuroregenerative effects. ${ }^{23}$ Preconditioning with TNF- $\alpha$ or endotoxin lipopolysaccharides will similarly attenuate the ischemic injury via an enhanced production of growth factors via the NF- $\kappa B$ pathway. ${ }^{24}$

\section{Cell-free therapy in preclinical ischemic stroke model}

MSCs exert their therapeutic efficacy partially via paracrine action. MSC secretomes exhibit diverse functions such as anti-inflammatory, immunomodulatory, anti-apoptotic, and angiogenic activities. MSCs were an ideal cellular therapy source for neurological disorders because of the secretion of several neurotrophic factors such as nerve growth factor, BDNF, and glial-derived neurotrophic factor. ${ }^{25}$

MSC-CM, referred to as MSC conditioned medium, has been shown to attenuate injury after stroke, improve functional recovery, reduce BBB leakage, and promote vascular remodeling in the ischemic brain. ${ }^{26} \mathrm{MSC}-\mathrm{CM}$ also promote astrocyte survival associated with GFAP and AQP4 downregulation, and the effects were most likely via p38 MAPK and JNK pathway. ${ }^{19,27}$ In the same model, MSC-CM derived from ischemic animals showed equal effectiveness as MSC$\mathrm{CM}$ derived from normal animals. Both of these remarkably improved neurological function, promoted neurogenesis, and attenuated microglia/macrophage infiltration. ${ }^{28}$

MSC secretome contains several groups of secreted vesicles, which mainly include exosomes, microvesicles, and apoptotic bodies. MSC secretome has unparalleled advantages in clinical application as the following: 1) MSCs are less immunogenic than cells owing to lower expression of membrane-bound proteins such as major histocompatibility complex molecules; 2) MSC exosomes can be stored without potentially toxic cryoprotectant at $-20^{\circ} \mathrm{C}$ for 6 months with no loss of their biochemical activity; ${ }^{29}$ and 3) MSC exosomes can pass through the BBB. ${ }^{30}$ Recent studies have proven the potential of exosomes as carriers for brain delivery of anticancer drugs for the treatment of brain cancer. ${ }^{31}$ Exosomes could transfer miRNAs which enhance tissue repair and functional recovery, and its potential as a therapeutic approach for stroke after MSCs therapy were also under discussion. ${ }^{32}$

\section{MSCs in clinical trials}

The therapeutic promise of MSCs in preclinical studies has initiated some early-phase clinical trials for ischemic stroke. MSCs derived from bone marrow, umbilical cord, adipose tissue, or serum transplanted at 30-150 days after stroke by means of intravenous/intra-arterial administration have shown promising results in early clinical trials. The neurological functions, daily living abilities, and disability levels of the patients, as evaluated with the National Institutes of Health Stroke Scale and the modified Rankin Scale, were improved. ${ }^{33-36}$ Moreover, during the 5-year follow-up, mortality due to any cause was lower, and the cumulative surviving portion at 260 weeks was significantly higher than the control group..$^{33}$

Recently, stereotactic administration was used in MSC treatment in stroke and has shown remarkable results. In the 2-year, open-label, single-arm, Phase I/IIa study, modified human bone marrow-derived MSCs (transient transfection with human Notch-1 intracellular gene) from healthy donors were implanted to 18 patients with chronic motor deficits between 6 and 60 months after sustaining an ischemic stroke, using magnetic resonance imaging stereotactic technique to restrict the target sites surrounding the residual stroke region. After 12 months of follow-up, significant improvement from baseline was reported for several neurological deficits scores, and the area of magnetic resonance $\mathrm{T} 2$ fluid in the ipsilateral cortex attenuated recovery signal change 1 week after implantation, which was significantly related to clinical improvement at 12 months. ${ }^{37}$

Many studies have proven the safety of MSC therapy. To date, there have been no studies reporting teratoma formation after the use of MSCs for treating ischemic stroke in long-term follow-up clinical trials. In trials with intravenous administration, most adverse effects appeared within the first 24 hours after MSC treatment, and they regressed spontaneously with no special treatment. No significant adverse effects were observed following transplantation..$^{33,34,36}$ Comorbidities including seizures and recurrent vascular episodes did not occur differently compared with the control group in 5-year follow-up. ${ }^{34}$ On intracerebral administration, none of the adverse effects observed were definitely related to cell treatment, and all serious treatment-emergent adverse events resolved without sequelae. ${ }^{37}$

\section{Challenges in clinical MSCs therapy}

Although allo-MSCs have been shown to have equivalent efficacy to auto-MSCs in stroke models, recent studies have found that allo-MSCs are not fully immune privileged as previously suggested. Several in vivo studies demonstrated that they do elicit a humoral and cellular immune response. Zangi et $a{ }^{38}$ found that luciferase-expressing mMSCs died by day 20 after being injected into allogeneic hosts. Moreover, 
allo-MSCs seem to induce immune memory. ${ }^{39}$ Others reported that allo-MSCs stimulated innate immune responses such as encouraging macrophage and neutrophil infiltration to the injection site. ${ }^{40}$ Different groups have shown inconsistent or even opposite outcomes of allo-MSCs therapy in various experiments. ${ }^{41}$ To sum up, although in vivo immunogenicity of MSCs needs a comprehensive understanding and remains to be determined, there is doubt that MSCs are immune evasive rather than immune privileged. It is critical to take immunogenicity and its impact on clinical utilization into consideration and recognize it as a feature of MSCs. In any case, use of allo-MSCs in clinical trials needs reappraisal. Selection of appropriate genes to modify MSCs may be a solution.

Not only do allo-MSCs face significant challenges, but auto-MSCs also have drawbacks. An ideal procedure meeting the human clinical demands of amount, quality, and short expansion time has not been created. Optimizing a protocol to generate hMSCs or their secretome should be put on agenda.

\section{Acknowledgments}

This project was supported by grants from the National Natural Sciences Foundation of China (No. JX4A03 and No. 81471201)

\section{Disclosure}

The authors report no conflicts of interest in this work.

\section{Reference}

1. Majid A. Neuroprotection in stroke: past, present, and future. ISRN Neurol. 2014;2014:515716.

2. Greco SJ, Corcoran KE, Cho KJ, Rameshwar P. Tachykinins in the emerging immune system: relevance to bone marrow homeostasis and maintenance of hematopoietic stem cells. Front Biosci. 2004;9:1782-1793.

3. Dominici M, Le Blanc K, Mueller I, et al. Minimal criteria for defining multipotent mesenchymal stromal cells. The International Society for Cellular Therapy position statement. Cytotherapy. 2006;8(4): $315-317$.

4. Trounson A, McDonald C. Stem cell therapies in clinical trials: progress and challenges. Cell Stem Cell. 2015;17(1):11-22.

5. Aleynik A, Gernavage KM, Mourad Y, et al. Stem cell delivery of therapies for brain disorders. Clin Transl Med. 2014;3:24.

6. Liu L, Eckert MA, Riazifar H, Kang DK, Agalliu D, Zhao W. From blood to the brain: can systemically transplanted mesenchymal stem cells cross the blood-brain barrier? Stem Cells Int. 2013;2013: 435093.

7. Zhao Y, Wang X, Dong P, et al. Bone marrow derived mesenchymal stem cells alleviated brain injury via down-regulation of interleukin1 beta in focal cerebral ischemic rats. Am J Transl Res. 2016;8(3): $1541-1550$.

8. Kim S, Chang KA, Kim J, et al. The preventive and therapeutic effects of intravenous human adipose-derived stem cells in Alzheimer's disease mice. PLoS One. 2012;7(9):e45757.

9. Pirzad Jahromi G, Shabanzadeh Pirsaraei A, Sadr SS, et al. Multipotent bone marrow stromal cell therapy promotes endogenous cell proliferation following ischemic stroke. Clin Exp Pharmacol Physiol. 2015; 42(11):1158-1167.
10. Li Y, Chen J, Chen XG, et al. Human marrow stromal cell therapy for stroke in rat: neurotrophins and functional recovery. Neurology. 2002;59(4):514-523.

11. Horita Y, Honmou O, Harada K, Houkin K, Hamada H, Kocsis JD. Intravenous administration of glial cell line-derived neurotrophic factor gene-modified human mesenchymal stem cells protects against injury in a cerebral ischemia model in the adult rat. $J$ Neurosci Res. 2006;84(7):1495-1504.

12. Chung TN, Kim JH, Choi BY, Chung SP, Kwon SW, Suh SW. Adipose-derived mesenchymal stem cells reduce neuronal death after transient global cerebral ischemia through prevention of blood-brain barrier disruption and endothelial damage. Stem cells Transl Med. 2015;4(2):178-185.

13. Moisan A, Favre I, Rome C, et al. Intravenous injection of clinical grade human MSCs after experimental stroke: functional benefit and microvascular effect. Cell Transplant. Epub 2016 Feb 26.

14. Zhang LL, Zhang HT, Cai YQ, et al. Anti-inflammatory effect of mesenchymal stromal cell transplantation and quercetin treatment in a rat model of experimental cerebral ischemia. Cell Mol Neurobiol. 2016;36(7):1023-1034.

15. Lee SH, Jin KS, Bang OY, et al. Differential migration of mesenchymal stem cells to ischemic regions after middle cerebral artery occlusion in rats. PLoS One. 2015;10(8):e0134920.

16. Zhang L, Li Y, Zhang C, Chopp M, Gosiewska A, Hong K. Delayed administration of human umbilical tissue-derived cells improved neurological functional recovery in a rodent model of focal ischemia. Stroke. 2011;42(5):1437-1444.

17. Shin TH, Phukan G, Shim JS, et al. Restoration of polyamine metabolic patterns in in vivo and in vitro model of ischemic stroke following human mesenchymal stem cell treatment. Stem Cells Int. 2016;2016:4612531.

18. Cheng $Q$, Zhang Z, Zhang S, et al. Human umbilical cord mesenchymal stem cells protect against ischemic brain injury in mouse by regulating peripheral immunoinflammation. Brain Res. 2015;1594:293-304.

19. Tang G, Liu Y, Zhang Z, et al. Mesenchymal stem cells maintain bloodbrain barrier integrity by inhibiting aquaporin- 4 upregulation after cerebral ischemia. Stem Cells. 2014;32(12):3150-3162.

20. Jeong CH, Kim SM, Lim JY, Ryu CH, Jun JA, Jeun SS. Mesenchymal stem cells expressing brain-derived neurotrophic factor enhance endogenous neurogenesis in an ischemic stroke model. BioMed Res Int. 2014;2014:129145.

21. Yu X, Chen D, Zhang Y, et al. Overexpression of CXCR4 in mesenchymal stem cells promotes migration, neuroprotection and angiogenesis in a rat model of stroke. J Neurol Sci. 2012;316(1-2):141-149.

22. Lu H, Liu X, Zhang N, et al. Neuroprotective effects of brain-derived neurotrophic factor and noggin-modified bone mesenchymal stem cells in focal cerebral ischemia in rats. J Stroke Cerebrovasc Dis. 2016; 25(2):410-418.

23. Wei N, Yu SP, Gu X, et al. Delayed intranasal delivery of hypoxicpreconditioned bone marrow mesenchymal stem cells enhanced cell homing and therapeutic benefits after ischemic stroke in mice. Cell Transplant. 2013;22(6):977-991.

24. Crisostomo PR, Wang Y, Markel TA, Wang M, Lahm T, Meldrum DR. Human mesenchymal stem cells stimulated by TNF-alpha, LPS, or hypoxia produce growth factors by an NF kappa B- but not JNK-dependent mechanism. Am J Physiol Cell Physiol. 2008;294(3): C675-C682.

25. Lopatina T, Kalinina N, Karagyaur M, et al. Adipose-derived stem cells stimulate regeneration of peripheral nerves: BDNF secreted by these cells promotes nerve healing and axon growth de novo. PLoS One. 2011; 6(3):e17899.

26. Zhao Q, Hu J, Xiang J, et al. Intranasal administration of human umbilical cord mesenchymal stem cells-conditioned medium enhances vascular remodeling after stroke. Brain Res. 2015;1624:489-496.

27. Huang W, Lv B, Zeng H, et al. Paracrine factors secreted by MSCs promote astrocyte survival associated with GFAP downregulation after ischemic stroke via p38 MAPK and JNK. J Cell Physiol. 2015;230(10):2461-2475. 
28. Tsai MJ, Tsai SK, Hu BR, et al. Recovery of neurological function of ischemic stroke by application of conditioned medium of bone marrow mesenchymal stem cells derived from normal and cerebral ischemia rats. J Biomed Sci. 2014;21:5.

29. Webber J, Clayton A. How pure are your vesicles? J Extracell Vesicles. 2013;2:1-6.

30. Zhang Y, Chopp M, Meng Y, et al. Effect of exosomes derived from multipluripotent mesenchymal stromal cells on functional recovery and neurovascular plasticity in rats after traumatic brain injury. J Neurosurg. 2015;122(4):856-867.

31. Yang T, Martin P, Fogarty B, et al. Exosome delivered anticancer drugs across the blood-brain barrier for brain cancer therapy in Danio rerio. Pharm Res. 2015;32(6):2003-2014.

32. Xin H, Li Y, Chopp M. Exosomes/miRNAs as mediating cell-based therapy of stroke. Front Cell Neurosci. 2014;8:377.

33. Jiang Y, Zhu W, Zhu J, Wu L, Xu G, Liu X. Feasibility of delivering mesenchymal stem cells via catheter to the proximal end of the lesion artery in patients with stroke in the territory of the middle cerebral artery. Cell Transplant. 2013;22(12):2291-2298.

34. Lee JS, Hong JM, Moon GJ, Lee PH, Ahn YH, Bang OY; STARTING collaborators. A long-term follow-up study of intravenous autologous mesenchymal stem cell transplantation in patients with ischemic stroke. Stem Cells. 2010;28(6):1099-1106.
35. Qiao LY, Huang FJ, Zhao M, et al. A two-year follow-up study of cotransplantation with neural stem/progenitor cells and mesenchymal stromal cells in ischemic stroke patients. Cell Transplant. 2014;23 (Suppl 1):S65-S72.

36. Honmou $\mathrm{O}$, Houkin $\mathrm{K}$, Matsunaga $\mathrm{T}$, et al. Intravenous administration of auto serum-expanded autologous mesenchymal stem cells in stroke. Brain. 2011;134(Pt 6):1790-1807.

37. Steinberg GK, Kondziolka D, Wechsler LR, et al. Clinical outcomes of transplanted modified bone marrow-derived mesenchymal stem cells in stroke: a phase 1/2a study. Stroke. 2016;47(7):1817-1824.

38. Zangi L, Margalit R, Reich-Zeliger S, et al. Direct imaging of immune rejection and memory induction by allogeneic mesenchymal stromal cells. Stem Cells. 2009;27(11):2865-2874.

39. Nauta AJ, Westerhuis G, Kruisselbrink AB, Lurvink EG, Willemze R, Fibbe WE. Donor-derived mesenchymal stem cells are immunogenic in an allogeneic host and stimulate donor graft rejection in a nonmyeloablative setting. Blood. 2006;108(6):2114-2120.

40. Grinnemo KH, Mansson A, Dellgren G, et al. Xenoreactivity and engraftment of human mesenchymal stem cells transplanted into infarcted rat myocardium. J Thorac Cardiovasc Surg. 2004;127(5):1293-1300.

41. Lee KC, Lin HC, Huang YH, Hung SC. Allo-transplantation of mesenchymal stem cells attenuates hepatic injury through IL1Ra dependent macrophage switch in a mouse model of liver disease. J Hepatol. 2015;63(6): 1405-1412.
Journal of Neurorestoratology

\section{Publish your work in this journal}

The Journal of Neurorestoratology is an international, peer-reviewed, open access online journal publishing original research and review articles on the subject of Neurorestoratology. To provide complete coverage of this revolutionary field the Journal of Neurorestoratology will report on relevant experimental research, technological advances,

\section{Dovepress}

and clinical achievements. The manuscript management system is completely online and includes a very quick and fair peer-review system, which is all easy to use. Visit http://www.dovepress.com/testimonials. php to read real quotes from published authors. 